\title{
A NOVEL DISTRIBUTED AND PRACTICAL INCENTIVE MECHANISM FOR PEER TO PEER LIVE VIDEO STREAMING
}

\author{
Yun Tang, Lifeng Sun, Meng Zhang, Shiqiang Yang, Yuzhuo Zhong \\ Department of Computer Science and Technology, Tsinghua University, Beijing 100084,P.R.China \\ tangyun98@mails.tinghua.edu.cn, sunlf@tsinghua.edu.cn, zhangmeng00@mails.tisnghua.edu.cn, \\ yangshq@tsinghua.edu.cn, zyz-dcs@tsinghua.edu.cn
}

\begin{abstract}
The successful deployment of peer-to-peer (P2P) live video streaming systems has practically demonstrated that it can scale to reliably support a large population of peers. However, peers, representing rational end users, tend to be noncooperative when it comes to the duty rather than the selfinterests, running counter to the fundamental design philosophy of P2P concept. The objective of this paper is to investigate the problem of encouraging users to balance what they take from the system with what they contribute. We first make a statistical analysis to the service logs of a practical $\mathrm{P} 2 \mathrm{P}$ live video streaming system and reveal intrinsic characteristic of users' online duration. Second, we thus propose a novel incentive mechanism based on the composite contributions which consist of two objective metrics, i.e. the online duration and effective upstream traffic. This mechanism offers service differentiation to users with different contributions and has some desirable properties: (1) distributed nature upon gossip-based overlay structure and (2) practical oriented evaluation criteria. The experiment results over PlanetLab further verify its effectiveness.
\end{abstract}

\section{INTRODUCTION}

With the explosive growth of Internet and information communication technologies in past years, provisioning data or service to a large population of users remains interesting and challenging. Among various research efforts, P2P network became the most perspective means since it exploited the cooperative paradigm of information exchange. Besides a variety of well-known file sharing and VoIP applications, the successful deployment service of our practical system also demonstrated that P2P networks can scale to reliably support large numbers of users for live video streaming [1].

Intrinsic to the success of those $\mathrm{P} 2 \mathrm{P}$ systems is clearly the cooperation between all participants. However, peers, representing rational users, tend to optimize towards their own outcomes without contributing (e.g. refusing forwarding video

This work is supported by the National Natural Science Foundation of China under Grant No.60432030 and No.60503063 segments or control packets which otherwise would consume the uploading bandwidth). When those non-cooperative users benefit from so-called "freeriding" on others [2], system performance degrades and the "tragedy of the commons" is inevitable [3]. Thus, incentive mechanism that encourages users to contribute their resources to the service community may be critical for the eventual success of $\mathrm{P} 2 \mathrm{P}$ applications. A rich body of previous literature discussed incentive mechanisms while apply to file sharing applications, comprising monetary payment, reputation, reciprocity and game theoretic approaches [4], [5], [6], [7].

As compared to well-studied file sharing applications,two particular characteristics distinguish the arena of live video streaming service, that is, the stringent playback deadline instead of file downloading integrity and high demanding bandwidth over heterogeneous underlay networks, which makes the design of incentive mechanism inherently different from those in the state of the art. In this context, the objective of this paper is to investigate following issues within $\mathrm{P} 2 \mathrm{P}$ live video streaming applications:

(1) From the microscopic view, how to effectively and objectively evaluate how much each user contributes into the system?

(2) From the macroscopic view, how to provide service differentiation to users with different contributions for global fairness?

We first make a statistical analysis to the service logs of our practical system and reveal that peers who had stayed in the community longer would be willing to spend more time. Then the statistically positive correlation motivate us to propose a novel incentive mechanism which is composed of two objective metrics, i.e. online duration and effective upstream traffic. Wherein the former is explored to measure the fidelity of peers while the later would be evaluated by the downstream partners within the horizon of playback deadline and utilized to encourage mutual cooperation between peers. The proposed incentive mechanism offers appealing fairness through peer selection based differentiated service. Peers which have already been active for a longer time and contributed more effective video segments are rewarded with 
better quality streaming service in return. Freeriders are limited to, if any, poor upstream nodes, resulting in low quality of streaming sessions. The experiment results over PlanetLab also confirm its effectiveness.

The balance of this paper is organized as follows. Sec.2 gives a brief overview to the practical live video streaming service and then provides statistical analysis to online duration of end users, which further motivates us to propose the incentive mechanism in Sec.3. Some preliminary experiment results are exhibited in Sec.4. Sec.5 discusses future work and ends this paper.

\section{LIVE VIDEO STREAMING SERVICE OVER INTERNET}

We have developed and implemented a P2P live video streaming system and provided broadcasting service over global Internet for numerous important events [1]. It is commonly believed that the practical experiences and fundamental observations could serve as a basis of design philosophy of $\mathrm{P} 2 \mathrm{P}$ architectures and related mechanisms. Therefore, we briefly describe the service overview in first subsection and then statistically analyze the online duration in subsection 2.2 to elicit the proposed incentive mechanism,.

\subsection{Overview}

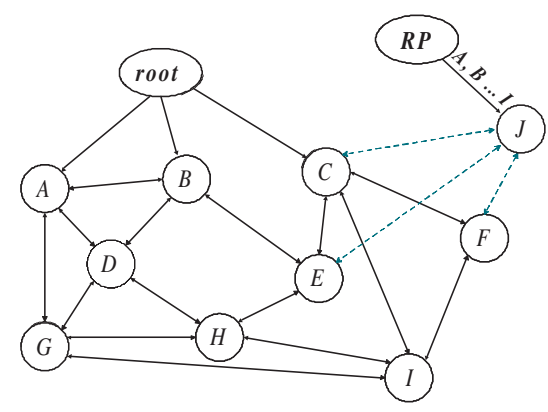

Fig. 1. A case of gossip based overlay structure

Since gossip-based overlay in general offers inherent resilience to accommodate high topology change in $\mathrm{P} 2 \mathrm{P}$ network [8], we also use it to perform distributed membership management in overlay construction. Fig.1 illustrates a case of gossip based overlay structure. Each peer takes charge of maintaining part of total members that are currently active in the service community. And everyone selects a proportion of nodes in the fractional member list as the upstream suppliers to fetch video contents. All those peers accordingly self organize into an overlay in a gossip fashion. Besides the member list and its evolution, each peer also refines its neighbor list in response to bidirectional traffic. The huge volume of streamed video date is transmitted among neighbors and eventually to the whole overlay network. As alternative to traditional client-server approach, this system was adopted by CCTV, the largest TV station in China, to live broadcast the Spring Festival Evening show over Internet in Feb. 2005. There were more than 500,000 users from about 66 countries over the world subscribing the service while the maximum number of concurrent users reached its peak 15,239 at Feb. 8th, 2005 More details about the system and deployment experiences could be referred to [1].

\subsection{Statistical analysis to online duration}

Inspired by the positive results of deployment experiences, we analyzed the service $\log$ s to reveal user characteristics, specifically with respect to online duration evolution of end users. Fig. 2 shows the cumulative distribution function of the online session duration. Observe that nearly $50 \%$ users spent less than 200 seconds in the community, which indicates that many peers would not stay for a longer while, resulting in extremely high churn rate. However, there are also roughly $20 \%$ users would like to keep online for more than 30 minutes.

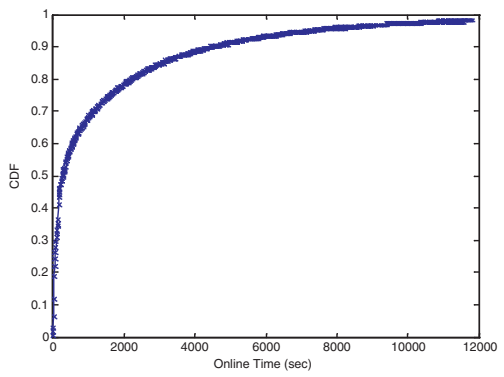

Fig. 2. CDF of online session duration

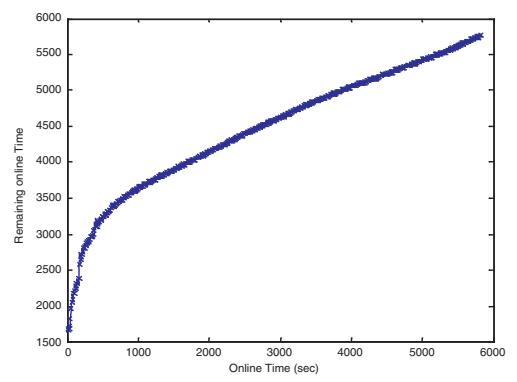

Fig. 3. Relation between average remaining online time and elapsed online time

Then we intentionally investigate the relation between elapsed online time and average remaining online time in Fig.3. It is 
obvious that peers who had stayed longer would be expected to spend more time to enjoy the service, as similar to that in client-server service. The main reason of this positive correlation may reside in the popularity-driven access pattern of live video programs, which is significantly different from the "fetch-at-most-once" mode of file sharing applications.

\section{DESIGN OF DISTRIBUTED AND PRACTICAL INCENTIVE MECHANISM}

Since most of existing work either sticks on file sharing applications or advocates intuitionistic criteria for contribution evaluation, we aim to address the issues posed previously with the benefit of above practical features: by contributing more and staying longer it is quite possible to improve the service of streaming quality one obtains in return. The design of incentive mechanism is naturally carried out in two steps. One is to effectively and objectively evaluate the contribution of a peer, while the other is to provide differentiated service to peers with different contribution levels.

Before we go into the details of this mechanism, let's first define the effective upstream traffic as the amount of packets transmitted by the upstream suppliers and arriving before the playback deadline. For the first question, we clearly recognize that only the volume of outgoing traffic measured by their own [9] is far from enough. Therefore, we tentatively introduce the composite contributions, consisting of two objective metrics-online duration and effective upstream trafficas the basis of the incentive mechanism. The former could be explored to measure the fidelity of peers, while the "effectiveness" in the later would be evaluated by the downstream partners within the horizon of playback deadline and utilized to encourage mutual cooperation among peers. The composite contributions essentially differentiate peers along these two dimensions. Formally speaking, if we denote $\delta$ as the freeriding tolerance factor, for each node $p$, the composite contribution could be calculated as

$$
C_{p}=\left\{\begin{array}{l}
\frac{C_{E T}}{\gamma C_{T}}, \frac{C_{E T}}{C_{T}}<\delta \\
\gamma_{1} C_{E T}+\gamma_{2} C_{T}, \frac{C_{E T}}{C_{T}} \geq \delta
\end{array}\right.
$$

where $C_{E T}$ denotes effective upstream traffic of $p, C_{T}$ denotes online duration and $\gamma, \gamma_{1}, \gamma_{2}$ are the normalized factors. Note that those who had enjoyed the service for a long time with less effective upstream traffic, in turn low cooperative cooperation level, would be perceived by the threshold $\delta$. As each peer subscribing the service is required to synchronize with RP in Fig.1, $C_{T}$ could be measured in following equation:

$$
C_{T}=T_{c u r}-T_{s y n_{p}}
$$

where $T_{c u r}$ is current time and $T_{s y n_{p}}$ is the synchronization time of node $p$. Since node might be involved in previous transactions with multiple peers, we could obtain the effective upstream traffic $C_{E T}$ as follows. For each peer $u$ who received effective packets $\left\{B_{p i}^{1}, B_{p i}^{2}, \ldots, B_{p i}^{n}\right\}$ in $n$ rounds, the effective upstream traffic between $i$ and $p$ could be calculated as:

$$
C_{B(p, i)}=\left\{\begin{array}{l}
\alpha^{n} B_{p i}^{1}+\alpha^{n-1} B_{p i}^{2}+\cdots+\alpha B_{p i}^{n}, n \neq 0 \\
0, n=0
\end{array}\right.
$$

Note that the exponential aggregation $(0<\alpha<1)$ here is used to highlight the most recent upstream traffic. Therefore, the aggregated effective upstream traffic of node $p$ could be objectively calculated as Eq. (4), where $m$ denotes the number of past transaction partners and $w_{i}$ is the weight of each partner:

$$
C_{E B}=\left\{\begin{array}{l}
\sum_{i=1}^{m} w_{i} * C_{B(p, i)}, m \neq 0 \\
0, m=0
\end{array}\right.
$$

In this way the composite contributions of each peer have been objectively calculated. It should be stressed that it is trustworthy to compute $C_{p}$ in this way due to the rationality of end users. There are two potential cheating behaviors of node $p$ 's partners: increasing the contributions or decreasing them. However, on one hand, when they are requesting segments from another peer afterwards, adding the rival $p$ 's contributions will damage their own benefits. On the other hand, depreciating $p$ will have negative impacts on current service quality since $p$ might be dropped by $p$ 's upstream nodes for lower contributions. Therefore, there is no incentive and motivation for $p$ 's partners to misreport the actual value of $p$ 's contributions.

For the second question, we attempt to provide service differentiation through contributions-based upstream peer selection. Roughly speaking, "fans"who have already been active for a longer while and transferred more effective media segments are rewarded with better upstream peers who may be also the "fans" and in turn higher quality streaming sessions. "Freeriders" are constrained to few opportunities to select first-rank candidate supplying nodes, certainly resulting in low streaming quality.

In addition to the contribution evaluation and service differentiation mechanism, there remains a key question that confronts the proposed incentive mechanism, that is, how to link the two components together to track the past behaviors for determining the present service quality. Most of existing work generally involves a trusted third party for records and query response, which would suffer from the failure of single point and in turn result in poor scalability.However, with the gossipbased overlay structure in consideration, we seek to collect, aggregate and distribute these contributions in a distributed fashion along with the membership maintenance. As discussed briefly in subsection 2.1, factional member lists are periodically exchanged within the community and thus the composite contributions are able to be attached. Before requesting or forwarding video segments, each peer could retrieve the corresponding contributions of others from member list and neighbor list to select upstream candidates and 
decide whether cooperate with its partners. Although it might be argued that it is not efficient enough and will induce a certain number of communication overhead, we insist that the gossip-based distributed incentive mechanism could perceive cheating-like collusion within bounded rounds and the overhead will be studied in next section.

\section{PERFORMANCE STUDY AND EVALUATION}

Suppose the node amount of the $\mathrm{P} 2 \mathrm{P}$ network is $N$, the number of each node' neighbors is $M$, and the total segment amount of live broadcasting stream is $Q$. We discuss the performance of our algorithm by analyzing the computation complexity of each node and communication overhead of the overlay network at first. For the contribution evaluation, the computation burden is to evaluate the contribution of upstream peer when receiving a segment, and hence the computation complexity is $O(Q)$. Note that the computation load on each node is unrelated with the network size $N$. Similarly, the communication overhead of the contribution evaluation is that each time one peer receives a segment, it updates the contributions of upstream peers and refine the corresponding member list and neighbor list. For the updated information, the communication overhead of the contribution evaluation is $O(Q * N)$.

We also conduct extensive experiments over the Planetlab testbed to evaluate our incentive mechanism. The prototype in [1] is deployed as the underlying streaming system and in total we utilize more than 500 machines as peers in the service community. Due to the page limitation, here we simply refer to the average delivery ratio as the representative metric. The delivery ratio of each peer could be calculated as the ratio of packets that arrive before the playback deadline to the total packets, which indicates the visual quality at end users. Fig.4 shows that the average delivery ratio is lower when there is no incentive mechanism. With the proposed incentive mechanism, rational peers are encouraged to stay longer and make more contributions, resulting in better stability of the overlay and more cooperation between peers, and in turn higher average delivery ratio.

\section{CONCLUSIONS AND FUTURE WORK}

In this paper, we mainly investigate the problem of encouraging users to balance what they take from the system with what they contribute in P2P live video streaming system since such applications rely on mutual cooperation between peers. Our contributions come in two-fold. Firstly, we make the statistical analysis to service logs of the practical system and reveals inherent characteristic of user online duration. Secondly, we propose a novel incentive mechanism which integrates online duration information with the effective upstream traffic of each peer. The proposed incentive mechanism is substantially distributed due to the gossip-based overlay structure construction and practical due to the objective contribution criteria.

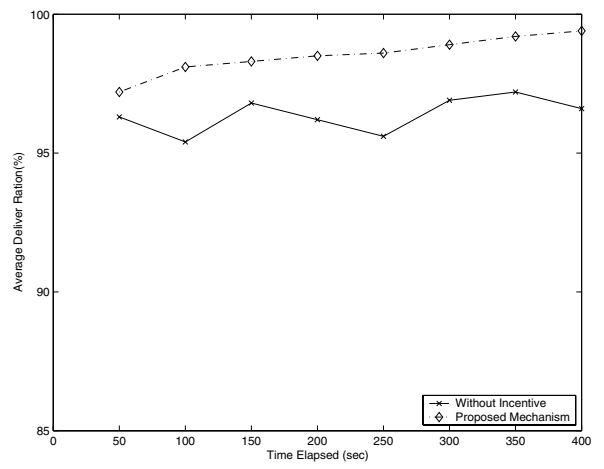

Fig. 4. Comparison of average delivery ration between with and without incentive mechanism

In the ongoing work, we will evaluate the overhead of proposed incentive mechanism and then deploy it into the practical system.

\section{REFERENCES}

[1] M. Zhang, L. Zhao, Y. Tang, J.G. Luo, S.Q. Yang. Large-Scale Live Media Streaming over Peer-to-Peer Networks through Global Internet. in Proceedings of P2MMS, ACM Multimedia 2005, November 2005.

[2] Adar E. and Huberman B.A., Free Riding on Gnutella. First Monday 5, 10. October 2000.

[3] Hardin G. The Tragedy of the Commons, Science 162(1968), 1243-1248.

[4] P. Golle, K.Leyton-Brown, and I. Mironov, Incentive for Sharing in Peer-to-Peer Networks, in Proceedings of ACM Electronic Commerce, Octorber 2001.

[5] M. Gupta, P. Judge, and M. Ammar, A Reputation System for Peer-to-Peer Networks, in Proceedings of ACM NOSSDAV, June 2003

[6] M. Nowak and K. Sigmund, Evolution of Indirect Reciprocity by Image Scoring, Nature, Vol. 393, pp. 573$577,1998$.

[7] Weihong Wang, Baochun Li. "To Play or to Control: A Game-based Control-theoretic Approach to Peer-toPeer Incentive Engineering," in Proceedings of IEEE International Workshop on Quality of Service, 2003.

[8] S. Banerjee, S. Lee, B. Bhattacharjee, A. Srinivasan. "Resilient multicast using overlays", In Proceedings of ACM SIGMETRICS 2004, June 2004.

[9] A. Habib, J. Chuang, Incentive Mechanism for Peer-toPeer Media Streaming, in Proceedings of IEEE International Workshop on Quality of Service, 2004. 